\title{
Periodicity, Stability, and Boundedness of Solutions to Certain Second Order Delay Differential Equations
}

\author{
A. T. Ademola, B. S. Ogundare, M. O. Ogundiran, and O. A. Adesina \\ Research Group in Differential Equations and Applications (RGDEA), Department of Mathematics, Obafemi Awolowo University, \\ Ile-Ife 220005, Nigeria
}

Correspondence should be addressed to O. A. Adesina; oadesina@oauife.edu.ng

Received 29 April 2016; Accepted 30 June 2016

Academic Editor: Elena Braverman

Copyright (C) 2016 A. T. Ademola et al. This is an open access article distributed under the Creative Commons Attribution License, which permits unrestricted use, distribution, and reproduction in any medium, provided the original work is properly cited.

The behaviour of solutions to certain second order nonlinear delay differential equations with variable deviating arguments is discussed. The main procedure lies in the properties of a complete Lyapunov functional which is used to obtain suitable criteria to guarantee existence of unique solutions that are periodic, uniformly asymptotically stable, and uniformly ultimately bounded. Obtained results are new and also complement related ones that have appeared in the literature. Moreover, examples are given to illustrate the feasibility and correctness of the main results.

\section{Introduction}

It is well known that the existence of boundedness, stability, and periodic solutions are among the most attractive topics in the qualitative behaviour of solutions to ordinary and functional differential equations. There are many articles dedicated so far to the qualitative properties of solutions of second order differential equations. Various authors have discussed these properties of solutions under numerous assumptions through different approaches. Some of these contributions include but are not limited to the works of Burton [1-3], Hale [4], and Yoshizawa [5-7] which contain general results on the subject matter and the expository papers of Ademola [8], Alaba and Ogundare [9], Cahlon and Schmidt [10], Domoshnitsky [11], Grigoryan [12], Jin and Zengrong [13], Kroopnick [14], Ogundare and Afuwape [15], Ogundare and Okecha [16], Tunç [17-21], Wang and Zhu [22], Xianfeng and Wei [23], and Yeniçerioğlu [24, 25] and the rich references cited therein.

In an interesting article, Yeniçerioğlu [24] considered the behavior of solutions of second order delay differential equation

$$
y^{\prime \prime}(t)=p_{1} y^{\prime}(t)+p_{2} y^{\prime}(t-\tau)+q_{1} y(t)+q_{2} y(t-\tau),
$$

where $p_{1}, p_{2}, q_{1}, q_{2}$ are real numbers and $\tau$ is positive real number and obtained criterion for stability of the considered equation. Furthermore, Yeniçerioğlu [25] discussed the stability properties of second order delay integrodifferential equations

$$
\begin{aligned}
x^{\prime \prime}(t)+a x(t) & =\int_{0}^{\tau} f(s) x(t-s) d s, \\
x(t) & =\phi(t),
\end{aligned}
$$

$$
-\tau \leq t \leq 0,
$$

where $a$ is a real number, $\tau$ is positive real number, $f$ is a continuous real-valued function on the interval $[0, \infty)$, and $\phi(t)$ is a given continuously differentiable initial function on the interval $[-\tau, 0]$.

In their contribution, Xianfeng and Wei [23] investigated the stability and boundedness of a retarded Lienard-type equation

$$
\begin{aligned}
x^{\prime \prime}+ & f_{1}(x) x^{\prime}+f_{2}(x) x^{\prime}(t-\tau)+f_{3}(x) x^{\prime 2}+\varphi(x) \\
& +g(x(t-\tau))=0,
\end{aligned}
$$

where $\tau$ is a nonnegative constant, $f_{1}, f_{2}, f_{3}$ and $\varphi$ are continuous functions on $\mathbb{R}$, and $g$ is differentiable on $\mathbb{R}$. 
For the case where there are no delay terms, we can mention some of the recent works of Alaba and Ogundare [9] and Ademola [8]. Alaba and Ogundare [9] studied the second order nonautonomous damped and forced nonlinear ordinary differential equation of the form

$$
\begin{aligned}
& {\left[a(t) x^{\prime}\right]^{\prime}+b(t) f\left(x, x^{\prime}\right) x^{\prime}+c(t) g(x)} \\
& \quad=p\left(t, x, x^{\prime}\right)
\end{aligned}
$$

where the functions $a, b, c, f, g$, and $p$ depend only on the arguments displayed explicitly. Another work worthy of mention in this regard is the article of Ademola [8] where the problem of stability, boundedness, and existence of periodic solutions of second order nonlinear nonautonomous ordinary differential equation

$$
\left[\phi(x) x^{\prime}\right]^{\prime}+g\left(t, x, x^{\prime}\right) x^{\prime}+\varphi(t) h(x)=p\left(t, x, x^{\prime}\right),
$$

where $\varphi: \mathbb{R}^{+} \rightarrow \mathbb{R}, \phi, h: \mathbb{R} \rightarrow \mathbb{R}$ and $g, p: \mathbb{R}^{+} \times \mathbb{R}^{2} \rightarrow \mathbb{R}$, was considered.

Motivated by the above discussions, in this paper, we consider the problem of uniform asymptotic stability, uniform ultimate boundedness, and existence of unique periodic solutions to a second order delay differential equation whose nonlinear functions contain variable deviating arguments. In effect, we consider

$$
\begin{aligned}
\ddot{x}(t)+ & \phi(t) f(x(t), x(t-\tau(t)), \dot{x}(t), \dot{x}(t-\tau(t))) \\
& +g(x(t-\tau(t)))=p(t, x(t), \dot{x}(t)),
\end{aligned}
$$

where $f, g, p$, and $\phi$ are continuous functions in their respective arguments on $\mathbb{R}^{4}, \mathbb{R}, \mathbb{R}^{+} \times \mathbb{R}^{2}$ and $\mathbb{R}^{+}$, respectively, with $\mathbb{R}=(-\infty, \infty)$ and $\mathbb{R}^{+}=[0, \infty)$. The continuity of these functions is sufficient for the existence of the solutions of (6). Furthermore, it is assumed that the functions $f, g$, and $p$ in (6) satisfy a Lipschitz condition in their respective arguments. The dots denote differentiation with respect to the independent variable $t$. If $\dot{x}(t)=y(t)$, then (6) is equivalent to the system

$$
\begin{aligned}
\dot{x}(t)= & y(t), \\
\dot{y}(t) & \\
= & p(t, x(t), y(t)) \\
& -\phi(t) f(x(t), x(t-\tau(t)), y(t), y(t-\tau(t))) \\
& -g(x(t))+\int_{t-\tau(t)}^{t} g^{\prime}(x(s)) y(s) d s,
\end{aligned}
$$

where $0 \leq \tau(t) \leq \alpha, \alpha>0$ is a constant to be determined later, and the derivative of the function $g$ (i.e., $g^{\prime}$ ) exists and is continuous for all $x$. To the best of our knowledge from the relevant literature, we are yet to come across any work with the above framework despite the practical importance of (6). This may not be unconnected with the practical difficulties associated with the construction of suitable complete
Lyapunov functional. Thus, the prime purpose of this paper is to fill the vacuum. An equally interesting problem is the situation where only the function $g$ in (6) is with a constant deviating argument. This has already been considered by us and the results arising in this direction will be advertized through another outlet.

The obtained results in the present paper are completely new and they extend previously known results in $[8,9]$ on second order ordinary differential equations and the works in [23-25] where the deviating arguments are constants. Some mathematical tools that will be needed in the sequel are discussed in Section 2. The main results of this paper are presented in Section 3 while examples are given in the last section to validate our results.

\section{Preliminary Results}

Consider the following general nonlinear nonautonomous delay differential equation:

$$
\begin{aligned}
\dot{X} & =\frac{d X}{d t}=F\left(t, X_{t}\right), \\
X_{t} & =X(t+\theta),
\end{aligned}
$$

$$
-r \leq \theta<0, t \geq 0
$$

where $F: \mathbb{R}^{+} \times C_{H} \rightarrow \mathbb{R}^{n}$ is a continuous mapping and $F(t+$ $\omega, \phi)=F(t, \phi)$ for all $\phi \in C$ and for some positive constant $\omega$. We assume that $F$ takes closed bounded sets into bounded sets in $\mathbb{R}^{n} .(C,\|\cdot\|)$ is the Banach space of continuous function $\varphi:[-r, 0] \rightarrow \mathbb{R}^{n}$ with supremum norm, $r>0$; for $H>0$, we define $C_{H} \subset C$ by $C_{H}=\{\varphi \in C:\|\varphi\|<H\} ; C_{H}$ is the open $H$-ball in $C, C=C\left([-r, 0], \mathbb{R}^{n}\right)$.

Definition 1 (see [3]). A continuous function $W: \mathbb{R}^{+} \rightarrow \mathbb{R}^{+}$ with $W(0)=0, W(s)>0$ if $s \neq 0$, and $W$ strictly increasing is a wedge. (We denote wedges by $W$ or $W_{i}$, where $i$ is an integer.)

Definition 2 (see [3]). The zero solution of (8) is asymptotically stable if it is stable and if for each $t_{0} \geq 0$ there is an $\eta>0$ such that $\|\phi\| \leq \eta$ implies that

$$
X\left(t, t_{0}, \phi\right) \longrightarrow 0 \quad \text { as } t \longrightarrow \infty \text {. }
$$

Definition 3 (see [2]). An element $\psi \in C_{H}$ is in the $\omega$-limit set of $\phi$, say $\Omega(\phi)$, if $X(t, 0, \phi)$ is defined on $\mathbb{R}^{+}$and there is a sequence $\left\{t_{n}\right\}, t_{n} \rightarrow \infty$ as $n \rightarrow+\infty$, with $\left\|X_{t_{n}}(\phi)-\psi\right\| \rightarrow 0$ as $n \rightarrow \infty$, where $X_{t_{n}}(\phi)=X\left(t_{n}+\theta, 0, \phi\right)$ for $-r \leq \theta<0$.

Definition 4 (see [26]). A set $Q \subset C_{H}$ is an invariant set if for any $\phi \in Q$, the solution $X(t, 0, \phi)$ of the system (8) is defined on $\mathbb{R}^{+}$, and $X_{t}(\phi) \in Q$ for $t \in \mathbb{R}^{+}$.

Lemma 5 (see [7]). Suppose that $F(t, \phi) \in \bar{C}_{0}(\phi)$ and $F(t, \phi)$ is periodic in tof period $\omega, \omega \geq r$, and consequently for any $\alpha>0$ there exists an $L(\alpha)>0$ such that $\phi \in C_{\alpha}$ implies $|F(t, \phi)| \leq$ $L(\alpha)$. Suppose that a continuous Lyapunov functional $V(t, \phi)$ exists, defined on $t \in \mathbb{R}^{+}, \phi \in S^{*}, S^{*}$ is the set of $\phi \in C$ such that 
$|\phi(0)| \geq H$ ( $H$ may be large $)$, and $V(t, \phi)$ satisfies the following conditions:

(i) $a(|\phi(0)|) \leq V(t, \phi) \leq b(\|\phi\|)$, where $a(r)$ and $b(r)$ are continuous, increasing, and positive for $r \geq H$ and $a(r) \rightarrow \infty$ as $r \rightarrow \infty$;

(ii) $\dot{V}_{(8)}(t, \phi) \leq-c(|\phi(0)|)$, where $c(r)$ is continuous and positive for $r \geq H$.

Suppose that there exists an $H_{1}>0, H_{1}>H$, such that

$$
h L\left(\gamma^{*}\right)<H_{1}-H \text {, }
$$

where $\gamma^{*}>0$ is a constant which is determined in the following way: By the condition on $V(t, \phi)$ there exist $\alpha>0, \beta>0$, and $\gamma>0$ such that $b\left(H_{1}\right) \leq a(\alpha), b(\alpha) \leq a(\beta)$, and $b(\beta) \leq$ $a(\gamma) . \gamma^{*}$ is defined by $b(\gamma) \leq a\left(\gamma^{*}\right)$. Under the above conditions, there exists a periodic solution of (8) of period $\omega$. In particular, inequality (10) can always be satisfied if h is sufficiently small.

Lemma 6 (see [7]). Suppose that $F(t, \phi)$ is defined and continuous on $0 \leq t \leq c, \phi \in C_{H}$ and that there exists a continuous Lyapunov functional $V(t, \phi, \varphi)$ defined on $0 \leq t \leq$ $c, \phi, \varphi \in C_{H}$ which satisfy the following conditions:

(i) $V(t, \phi, \varphi)=0$ if $\phi=\varphi$;

(ii) $V(t, \phi, \varphi)>0$ if $\phi \neq \varphi$;

(iii) for the associated system

$$
\begin{aligned}
& \dot{x}(t)=F\left(t, x_{t}\right), \\
& \dot{y}(t)=F\left(t, y_{t}\right)
\end{aligned}
$$

one has $V_{(11)}^{\prime}(t, \phi, \varphi) \leq 0$, where for $\|\phi\|=H$ or $\|\varphi\|=$ $H$, one understands that the condition $V_{(11)}^{\prime}(t, \phi, \varphi) \leq 0$ is satisfied in the case $V^{\prime}$ can be defined.

Then, for given initial value $\phi \in C_{H_{1}}, H_{1}<H$, there exists a unique solution of system (8).

Lemma 7 (see [7]). Suppose that a continuous Lyapunov functional $V(t, \phi)$ exists, defined on $t \in \mathbb{R}^{+},\|\phi\|<H, 0<$ $H_{1}<H$ which satisfies the following conditions:

(i) $a(\|\phi\|) \leq V(t, \phi) \leq b(\|\phi\|)$, where $a(r)$ and $b(r)$ are continuous, increasing, and positive;

(ii) $\dot{V}_{(8)}(t, \phi) \leq-c(\|\phi\|)$, where $c(r)$ is continuous and positive for $r \geq 0$.

Then the zero solution of the system (8) is uniformly asymptotically stable.

Lemma 8 (see [2]). Let $V: \mathbb{R}^{+} \times C \rightarrow \mathbb{R}$ be continuous and locally Lipschitz in $\phi$. If

(i) $W_{0}\left(\left|X_{t}\right|\right) \leq V\left(t, X_{t}\right) \leq W_{1}\left(\left|X_{t}\right|\right)+$ $W_{2}\left(\int_{t-r(t)}^{t} W_{3}\left(X_{t}(s)\right) d s\right)$

(ii) $\dot{V}_{(8)}\left(t, X_{t}\right) \leq-W_{4}\left(\left|X_{t}\right|\right)+N$, for some $N>0$ where $W_{i}(i=0,1,2,3,4)$ are wedges,

then $X_{t}$ of system (8) is uniformly bounded and uniformly ultimately bounded for bound $B$.

\section{Main Results}

We will give the following notations before we state our main results. Let $x(t)=x, y(t)=y$, and

$$
f(x, x(t-\tau(t)), y, y(t-\tau(t)))=f(\cdot) .
$$

Let $\left(x_{t}, y_{t}\right)$ be any solution of system (7); the main tool to proofs of our results is the continuously differentiable functional $V=V\left(x_{t}, y_{t}\right)$ defined as

$$
\begin{aligned}
2 V= & b\left(b x^{2}+y^{2}\right)+(a x+y)^{2}+2 \int_{0}^{x} g(s) d s \\
& +\int_{-\tau(t)}^{0} \int_{t+s}^{t}\left(\lambda_{1} x^{2}+\lambda_{2} y^{2}\right) d s d \theta,
\end{aligned}
$$

where $\lambda_{j}, j=1,2$, are constants to be determined. Next, we present the main results of this work.

Theorem 9. In addition to the assumptions on the functions $f, g, p$, and $\phi$, suppose that $a, b, \phi_{0}, B, \alpha, \beta, L, M$ are positive constants and that for all $t \geq 0$

(i) $a \leq f(\cdot) / y$ for all $x$ and $y \neq 0$;

(ii) $b \leq g(x) / x \leq B$ for all $x \neq 0$;

(iii) $\left|g^{\prime}(x)\right| \leq L$ for all $x$;

(iv) $1<\phi_{0} \leq \phi(t)$ for all $t \geq 0$;

(v) $0 \leq \tau(t) \leq \alpha, \tau^{\prime}(t) \leq \beta, 0<\beta<1$, where

$$
\begin{gathered}
\alpha<\min \left\{\frac{a b(1-\beta)}{L[a(1-\beta)+a+b+1]},\right. \\
\left.\frac{\left[a \phi_{0}(b+2)-2 a\right](1-\beta)}{L[(b+1)(1-\beta)+a+b+1]}\right\} ;
\end{gathered}
$$

(vi) $|p(t, x, y)| \leq M, 0<M<\infty$.

Then the solutions of the system (7) are uniformly bounded and uniformly ultimately bounded.

Remark 10. Below are some observations from previous results which the current results are addressing:

(i) When $\phi(t) f(\cdot)=a \dot{x}, g(x(t-\tau(t)))=b x$ and $p(t, x, y)=0$, then system (7) reduces to linear constant coefficients differential equation $\ddot{x}+a \dot{x}+b x=$ 0 , and conditions (i) to (vi) of Theorem 9 specialize to the corresponding Routh Hurwitz criteria $a>0$ and $b>0$.

(ii) When $\phi(t) f(\cdot)=c x^{\prime}, g(x(t-\tau(t)))=g(t, x)$, and $p(t, x, y)=h(t)$, system (7) specializes to ordinary differential equation discussed in [22]. Our result includes and extends the results in [22].

(iii) If $\tau(t)=\tau$ (a constant), $\phi(t) f(\cdot)=p_{1} y^{\prime}(t)+p_{2} y^{\prime}(t-\tau)$, and $g(x(t-\tau(t)))=q_{1} y(t)+q_{2} y(t-\tau)$, system (7) is trimmed down to that discussed in $[10,24]$. Thus our results improve the results in $[10,24]$. 
(iv) If $\tau(t)=0, \phi(t) f(\cdot)=c\left(t, x, x^{\prime}\right), g(x(t-\tau(t)))=$ $q(t) b(x)$, and $p(t, x, y)=f(t)$ then system $(7)$ reduces to that considered in [17]. Thus the result in [17] is a special case of Theorem 9.

(v) Whenever $\tau(t)=0,(6)$ reduces to the cases discussed in $[8,9]$.

In what follows, our purpose now is to state and prove the following result that would be used in the proofs of Theorem 9 and the subsequent ones.

Lemma 11. Under the hypotheses of Theorem 9, there exist positive constants $D_{0}=D_{0}(b, 1), D_{1}=D_{1}(a, b, B)$, and $D_{2}=D_{2}\left(\lambda_{1}, \lambda_{2}\right)$ such that

$$
\begin{aligned}
& D_{0}\left(x^{2}(t)+y^{2}(t)\right) \leq V\left(x_{t}, y_{t}\right) \\
& \quad \leq D_{1}\left(x^{2}(t)+y^{2}(t)\right) \\
& \quad+D_{2} \int_{t-\tau(t)}^{t}\left(x^{2}(s)+y^{2}(s)\right) d s,
\end{aligned}
$$

for all $t \geq 0, x$ and $y$.

Furthermore, there exist positive constants $D_{3}=$ $D_{3}\left(a, b, \phi_{0}, \alpha, \beta, L, 1\right)$ and $D_{4}=D_{4}(a, b, 1)$ such that

$$
\begin{aligned}
\dot{V}_{(7)}= & \left.\frac{d}{d t} V\left(x_{t}, y_{t}\right)\right|_{(7)} \\
= & -D_{3}\left(x^{2}(t)+y^{2}(t)\right) \\
& +D_{4}(|x(t)|+|y(t)|)|p(t, x(t), y(t))|,
\end{aligned}
$$

for all $t \geq 0, x$ and $y$.

Proof. Let $\left(x_{t}, y_{t}\right)$ be any solution of system (7). It is clear from (13) that $V(0,0)=0$. Since the double integrals terms are nonnegative, $g(x) \geq b x$ for all $x \neq 0$, and $(a x+y)^{2} \geq 0$ for all $x$ and $y$, then there exists a positive constant $\delta_{0}$ such that

$$
V \geq \delta_{0}\left(x^{2}+y^{2}\right),
$$

$t \geq 0, x$ and $y$, where

$$
\delta_{0}:=\frac{b}{2} .
$$

Thus the lower inequality in (15) holds with $\delta_{0}=D_{0}$. Also, from inequality (17) we deduced that

$$
\begin{aligned}
& V\left(x_{t}, y_{t}\right)=0 \Longleftrightarrow x^{2}+y^{2}=0 ; \\
& V\left(x_{t}, y_{t}\right)>0 \Longleftrightarrow x^{2}+y^{2} \neq 0, \\
& V\left(x_{t}, y_{t}\right) \longrightarrow+\infty \quad \text { as } x^{2}+y^{2} \longrightarrow \infty .
\end{aligned}
$$

Furthermore, since $g(x) \leq B x$ for all $x \neq 0$, and the fact that $2 x_{1} x_{2} \leq x_{1}^{2}+x_{2}^{2}$, then it follows from (13) that there exist positive constants $\delta_{1}$ and $\delta_{2}$ such that

$$
V \leq \delta_{1}\left(x^{2}+y^{2}\right)+\delta_{2} \int_{t-\tau(t)}^{t}\left(x^{2}(s)+y^{2}(s)\right) d s,
$$

for all $t \geq 0, x$ and $y$, where

$$
\begin{aligned}
& \delta_{1}=\frac{1}{2} \max \left\{a^{2}+b^{2}+a+B, a+b+1\right\}, \\
& \delta_{2}:=\frac{1}{2} \max \left\{\lambda_{1}, \lambda_{2}\right\} .
\end{aligned}
$$

Inequality (22) establishes the upper inequality in (15) where $\delta_{1}=D_{1}$ and $\delta_{2}=D_{2}$. Hence from inequalities (17) and (22), inequality (15) is established.

Next, let $\left(x_{t}, y_{t}\right)$ be any solution of the system (7). The time derivative of the function $V$, defined in (13), along a solution path of the system (7) is

$$
\begin{aligned}
\dot{V}_{(8)} & \\
= & -a \frac{g(x)}{x} x^{2}-\left[(b+1) \phi(t) \frac{f(\cdot)}{y}-a\right] y^{2} \\
& -\left\{a\left[\phi(t) \frac{f(\cdot)}{y}-a\right]+b\left[\frac{g(x)}{x}-b\right]\right\} x y \\
& +[a x+(b+1) y] p(t, x, y) \\
& +[a x+(b+1) y] \int_{t-\tau(t)}^{t} g^{\prime}(x(s)) y(s) d s \\
& +\frac{\tau(t)}{2}\left[\lambda_{1} x^{2}+\lambda_{2} y^{2}\right] \\
& -\frac{1}{2}\left(1-\tau^{\prime}(t)\right) \int_{t-\tau(t)}^{t}\left[\lambda_{1} x^{2}(\theta)+\lambda_{2} y^{2}(\theta)\right] d \theta .
\end{aligned}
$$

Now, from the hypotheses of Theorem $9, g(x) \geq b x$ for all $x \neq 0, f(\cdot) \geq a y$ for all $x$ and $y \neq 0, \phi(t) \geq \phi_{0}>1$ for all $t \geq 0,\left|g^{\prime}(x)\right| \leq L$ for all $x$, and the fact that $2 x_{1} x_{2} \leq x_{1}^{2}+x_{2}^{2}$, then from (24) it follows that

$$
\begin{aligned}
\dot{V}_{(8)} & \\
\leq & -a b x^{2}-\left[\frac{1}{2} a b \phi_{0}+a\left(\phi_{0}-1\right)\right] y^{2} \\
& +\max \{a, b+1\}(|x|+|y|)|p(t, x, y)| \\
& +\frac{L}{2}\left[a x^{2}+(b+1) y^{2}\right] \tau(t) \\
& +\frac{L}{2}(a+b+1) \int_{t-\tau(t)}^{t}\left[x^{2}(s)+y^{2}(s)\right] d s \\
& -\frac{1}{2}\left(1-\tau^{\prime}(t)\right) \int_{t-\tau(t)}^{t}\left[\lambda_{1} x^{2}(\theta)+\lambda_{2} y^{2}(\theta)\right] d \theta \\
& +\frac{1}{2}\left[\lambda_{1} x^{2}+\lambda_{2} y^{2}\right] \tau(t)-\sum_{j=1}^{2} W_{j},
\end{aligned}
$$

where

$$
\begin{aligned}
& W_{1}:=\frac{1}{4} a b x^{2}+a\left[\phi(t) \frac{f(\cdot)}{y}-a\right] x y+\frac{1}{4} \phi_{0} a b y^{2}, \\
& W_{2}:=\frac{1}{4} a b x^{2}+b\left[\frac{g(x)}{x}-b\right] x y+\frac{1}{4} \phi_{0} a b y^{2} .
\end{aligned}
$$


Employing the inequalities

$$
\begin{array}{r}
16 a^{-2}\left[\frac{g(x)}{x}-b\right]^{2}<\phi_{0}, \\
16 a^{-2}\left[\phi(t) \frac{f(\cdot)}{y}-a\right]^{2}<\phi_{0},
\end{array}
$$

for all $x \neq 0$ and $y \neq 0$, respectively, it follows that

$$
W_{1}=W_{2} \geq \frac{a b}{4}\left[|x|-\sqrt{\phi_{0}}|y|\right]^{2}>0 \text {, }
$$

$$
\forall t \geq 0,(x, y) \in \mathbb{R}^{2} .
$$

From inequality (28) and the fact that $0 \leq \tau(t) \leq \alpha$ and $\tau(t)^{\prime} \leq$ $\beta$ for all $t \geq 0$, the inequality in (25) yields

$$
\begin{aligned}
\dot{V}_{(8)} & \\
\leq & -\frac{1}{2} a b x^{2}-\left[\frac{1}{2} a b \phi_{0}+a\left(\phi_{0}-1\right)\right] y^{2} \\
& +\max \{a, b+1\}(|x|+|y|)|p(t, x, y)| \\
& +\frac{\alpha}{2}\left[(b+1) L+\lambda_{2}\right] y^{2} \\
& -\frac{1}{2}\left[\lambda_{1}(1-\beta)-(a+b+1) L\right] \int_{t-\tau(t)}^{t} x^{2}(\zeta) d \zeta \\
& +\frac{\alpha}{2}\left(a L+\lambda_{1}\right) x^{2} \\
& -\frac{1}{2}\left[\lambda_{2}(1-\beta)-(a+b+1) L\right] \int_{t-\tau(t)}^{t} y^{2}(\zeta) d \zeta .
\end{aligned}
$$

If we choose

$$
\lambda_{1}=\lambda_{2}=(1-\beta)^{-1}(a+b+1) L>0,
$$

and in view of inequality (14), there exist positive constants $\delta_{3}$ and $\delta_{4}$ such that inequality (29) becomes

$$
\dot{V}_{(8)} \leq-\delta_{3}\left(x^{2}+y^{2}\right)+\delta_{4}(|x|+|y|)|p(t, x, y)|,
$$

for all $t \geq 0, x$ and $y$, where

$$
\begin{aligned}
\delta_{3} & :=\frac{1}{2} \min \{a b \\
& -\left[a L+(1-\beta)^{-1}(a+b+1) L\right] \alpha, \phi_{0} a(b+2) \\
& \left.-2 a-\left[(b+1) L+(1-\beta)^{-1}(a+b+1) L\right] \alpha\right\}, \\
\delta_{4} & :=\max \{a, b+1\} .
\end{aligned}
$$

Estimate (31) establishes the inequality in (16) with $\delta_{3}$ and $\delta_{4}$ equal to $D_{3}$ and $D_{4}$, respectively. This completes the proof of Lemma 11.

Next, we will give the proof of Theorem 9.
Proof of Theorem 9. Let $\left(x_{t}, y_{t}\right)$ be any solution of system (7), hypothesis (vi) of Theorem 9 , and the fact that $|x|<1+x^{2}$, and then there exist positive constants $\delta_{5}$ and $\delta_{6}$ such that inequality (31) yields

$$
\dot{V}_{(8)} \leq-\delta_{5}\left(x^{2}+y^{2}\right)+\delta_{6},
$$

for all $t \geq 0, x$ and $y$, where $\delta_{5}:=\delta_{3}-\delta_{4} M>0$ and $\delta_{6}:=2 \delta_{4} M>0$. From inequalities (17), (22), and (33) the hypotheses of Lemma 8 hold. Thus by Lemma 8 , the solution $\left(x_{t}, y_{t}\right)$ of the system (7) is uniformly bounded and uniformly ultimately bounded. This completes the proof of Theorem 9.

Next, suppose that $p(t, x, \dot{x})=0$ and $p(t, x, y)=0$ in (6) and system (7), respectively; we have the following delay differential equation:

$$
\ddot{x}+\phi(t) f(\cdot)+g(x(t-\tau(t)))=0 .
$$

If $\dot{x}=y$, (34) is equivalent to the system

$$
\begin{aligned}
& \dot{x}=y, \\
& \dot{y}=-\phi(t) f(\cdot)-g(x)+\int_{t-\tau(t)}^{t} g^{\prime}(x(s)) y(s) d s,
\end{aligned}
$$

where the functions $f, g$, and $\phi$ are as defined in Section 1 . We have the following result for system (35).

Theorem 12. Suppose that hypotheses (i) to (v) of Theorem 9 hold; then the zero solution of system (35) is uniformly asymptotically stable.

Remark 13. (i) The stability results in $[2,8-10]$ are special cases of Theorem 12 .

(ii) When $\phi(t) f(\cdot)=p(t) \phi^{\prime}(t)$ and $g(x(t-\tau(t)))=$ $q(t) \phi(t)$ (34) reduces to the one discussed in [12].

(iii) Whenever $\phi(t) f(\cdot)=c x^{\prime}$ and $g(x(t-\tau(t)))=g(t, x)$ then (34) is a case studied in [22].

(iv) If $\phi(t) f(\cdot)=0$ and $g(x(t-\tau(t)))=a x(t)$ then (34) reduces to the one discussed in [25].

Proof of Theorem 12. Let $\left(x_{t}, y_{t}\right)$ be any solution of system (35), with hypotheses of Theorem 12; estimates (17), (21), and (22) hold. Furthermore, the derivative of the functional $V$ defined in (13) with respect to the independent variable $t$ along the solution path of the system (35) is

$$
\dot{V}_{(35)} \leq-\delta_{3}\left(x^{2}+y^{2}\right),
$$

for all $t \geq 0, x$ and $y$, where $\delta_{3}$ is defined after the inequality in (31). From inequalities (17), (21), (22), and (36) all hypotheses of Lemma 7 hold. Thus by Lemma 7 the zero solution of system (35) is uniformly asymptotically stable.

Theorem 14. If hypotheses (i) to (vi) of Theorem 9 hold, then there exists a unique periodic solution of period $\omega$ for the system (7). 
Proof. Let $\left(x_{t}, y_{t}\right)$ be any solution of system (7). In view of the hypotheses of Theorem 14 (19) and inequality (20) hold. Moreover, from hypothesis (vi) and the fact that

$$
|x|+|y| \leq 2^{1 / 2}\left(x^{2}+y^{2}\right)^{1 / 2}
$$

for all $x$ and $y$, it follows from inequality (31) that

$$
\dot{V}_{(7)} \leq-\delta_{3}\left(x^{2}+y^{2}\right)+2^{1 / 2} \delta_{4} M\left(x^{2}+y^{2}\right)^{1 / 2}
$$

$t \geq 0, x$ and $y$. Choose

$$
\left(x^{2}+y^{2}\right)^{1 / 2} \geq 2^{3 / 2} \delta_{3}^{-1} \delta_{4}
$$

and then there exists a positive constant $\delta_{7}$ such that

$$
\dot{V}_{(7)} \leq-\delta_{7}\left(x^{2}+y^{2}\right) \leq 0
$$

for all $t \geq 0, x$ and $y$, where $\delta_{7}:=(1 / 2) \delta_{3}$. Now from (19) and inequalities (20) and (40), all hypotheses of Lemma 7 hold. By Lemma 7, the existence of a unique solution for the system (7) is assured. Furthermore, from inequalities (17),
(21), (22), and (40) all hypotheses of Lemma 6 hold. Hence by Lemma 6 the existing unique solution is periodic of period $\omega$. This completes the proof of Theorem 14.

\section{Examples}

In this section, we present examples to check the validity and effectiveness of our results.

Example 1. Consider a second order delay differential equation

$$
\begin{aligned}
\ddot{x}+ & \left(1+\frac{1+\cos 3 t}{1+t^{2}}\right) \\
& +\left[1+\frac{\cos (x(t-\tau(t))) \cos (\dot{x}(t-\tau(t)))}{1+x^{2}(t-\tau(t))+\dot{x}^{2}(t-\tau(t))}\right] \dot{x} \\
& +\left[2+\frac{1 / 2+\sin (x(t-\tau(t)))}{1+x^{2}(t-\tau(t))}\right] x(t-\tau(t)) \\
& =\left(\frac{3+2 t+3 x^{2}+\dot{x}^{2}}{2\left[1+2 t+3 x^{2}+\dot{x}^{2}\right]}\right) .
\end{aligned}
$$

Equation (41) is equivalent to system of first order delay differential equation

$$
\begin{aligned}
\dot{x}= & y \\
\dot{y}= & \left(\frac{1}{2}+\frac{1}{1+2 t+3 x^{2}+y^{2}}\right)-\left[2+\frac{1 / 2+\sin x}{1+x^{2}}\right] x-\left(1+\frac{1+\cos 3 t}{1+t^{2}}\right)\left[1+\frac{\cos (x(t-\tau(t))) \cos (y(t-\tau(t)))}{1+x^{2}(t-\tau(t))+y^{2}(t-\tau(t))}\right] y \\
& +\int_{t-\tau(t)}^{t}\left[\frac{2 x(s)\left[x^{2}(s)+1\right] \cos x(s)+2\left[1-x^{2}(s)\right] \sin x(s)+4 x^{4}(s)+7 x^{2}(s)+5}{2\left[x^{2}(s)+1\right]^{2}}\right] d s .
\end{aligned}
$$

Comparing system (7) with (42), we obtain the following relations.

(i) The function

$$
\phi(t):=1+\frac{1+\cos 3 t}{1+t^{2}} .
$$

Since

$$
\frac{1+\cos 3 t}{1+t^{2}} \geq 2
$$

for all $t \geq 0$, it follows that

$$
\phi(t)=1+\frac{1+\cos 3 t}{1+t^{2}} \geq 3=\phi_{0}>1
$$

for all $t \geq 0$. The behaviour of $\phi(t)$ is shown in Figure 1 . (ii) The function

$$
f(\cdot):=\left[1+\frac{\cos (x(t-\tau(t))) \cos (y(t-\tau(t)))}{1+x^{2}(t-\tau(t))+y^{2}(t-\tau(t))}\right] y .
$$

It is not difficult to show that

$$
\begin{aligned}
\frac{f(\cdot)}{y} & =1+\frac{\cos (x(t-\tau(t))) \cos (y(t-\tau(t)))}{1+x^{2}(t-\tau(t))+y^{2}(t-\tau(t))} \geq 2 \\
& =a>0
\end{aligned}
$$

for all $t \geq 0, x$ and $y \neq 0$. See the behaviour of $f(\cdot)$ in Figure 2.

(iii) The function

$$
g(x):=\left[2+\frac{1 / 2+\sin x}{1+x^{2}}\right] x .
$$

Since

$$
-1 \leq \frac{1 / 2+\sin x}{1+x^{2}} \leq 1
$$

for all $x$, then we assert that

$$
1=b \leq \frac{g(x)}{x} \leq B=3, \quad \forall x \neq 0 .
$$




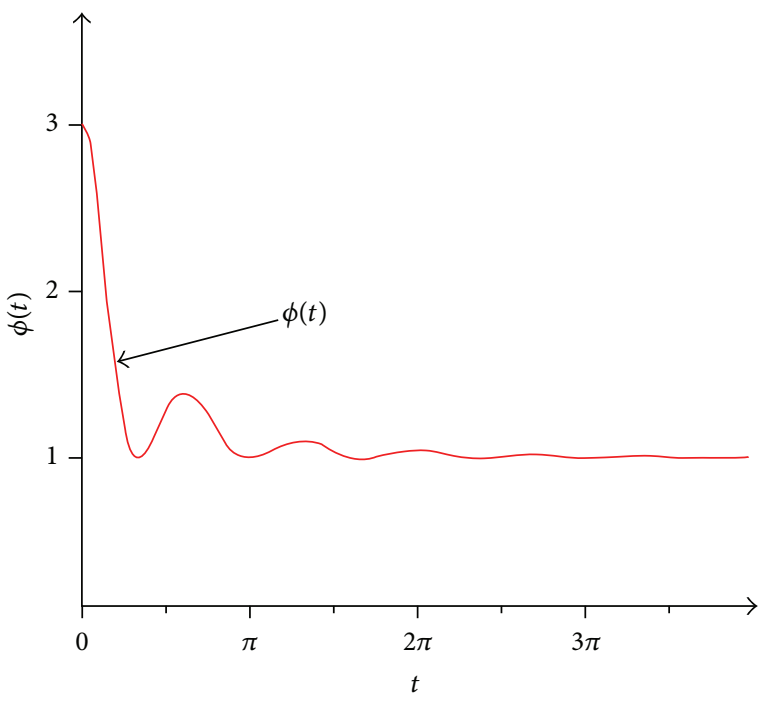

Figure 1: Functions $\phi(t)$.

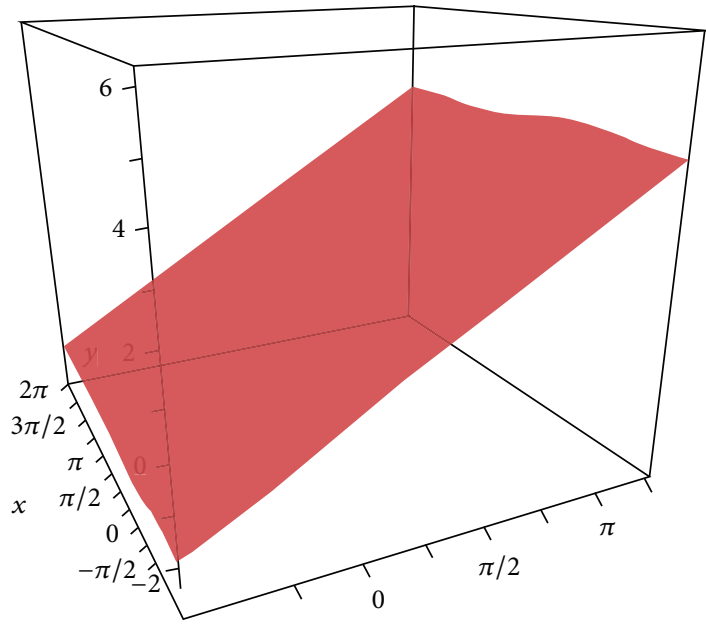

FIgURE 2: Functions $f(\cdot)$.

(iv) The function

$$
\begin{aligned}
& g^{\prime}(x) \\
& :=\frac{2 x\left[x^{2}+1\right] \cos x+2\left[1-x^{2}\right] \sin x+4 x^{4}+7 x^{2}+5}{2\left[x^{2}+1\right]^{2}} .
\end{aligned}
$$

Clearly, $g^{\prime}(x)$ is a bounded function; thus it is not difficult to show that

$$
\left|g^{\prime}(x)\right| \leq 3=L
$$

The boundedness of $g$ and $g^{\prime}$ is shown in Figure 3 .

(v) The function

$$
p(t, x, y):=\frac{1}{2}+\frac{1}{1+2 t+3 x^{2}+y^{2}} .
$$

Since

$$
\frac{1}{1+2 t+3 x^{2}+y^{2}} \leq 1
$$

for all $t \geq 0, x$ and $y$, it follows that

$$
|p(t, x, y)| \leq \frac{3}{2}=M<\infty
$$

for all $t \geq 0, x$ and $y$; and if

(vi) $\beta=1 / 2$, and with the values of $a, b, B, L, M$ as above, then inequality (14) becomes

$$
0<\alpha=\min \left\{\frac{1}{15}, \frac{7}{15}\right\}=\frac{1}{15} \text {. }
$$

All hypotheses of Theorems 9, 12, and 14 are satisfied; hence the conclusions of Theorems 9, 12, and 14 follow.

Example 2. Consider the second order delay differential equation

$$
\begin{aligned}
\ddot{x}+ & \left(\frac{5+2 t+\sin 6 t}{2+t^{2}}\right)(3+Q(x, \dot{x})) \dot{x} \\
& +[P(x)] x(t-\tau(t)) \\
= & \frac{1+x^{2}+\dot{x}^{2}+2 \cos (x+\dot{x})}{2\left[1+x^{2}+\dot{x}^{2}\right]},
\end{aligned}
$$

where

$$
\begin{aligned}
& Q(x, \dot{x}) \\
& :=\frac{\cos x+\cos (x(t-\tau(t)))+1 / 2 \cos \dot{x}+1 / 2 \cos (\dot{x}(t-\tau(t)))}{5+\cos ^{2} x+\cos ^{2} \dot{x}+x^{2}(t-\tau(t))+\dot{x}^{2}(t-\tau(t))}, \\
& P(x):=\frac{4+18 x(t-\tau(t))+\cos (3 x(t-\tau(t)))}{2+9 x^{2}(t-\tau(t))} .
\end{aligned}
$$

Equation (57) is equivalent to system of first order differential equation

$$
\begin{aligned}
\dot{x}= & y, \\
\dot{y}= & \frac{1+x^{2}+y^{2}+2 \cos (x+y)}{2\left[1+x^{2}+y^{2}\right]} \\
& -\left(\frac{5+2 t+\sin 6 t}{2+t^{2}}\right)(3+Q(x, y)) y \\
& -\left(\frac{4 x+18 x^{3}+x \cos 3 x}{2+9 x^{2}}\right) \\
& +\int_{t-\tau(t)}^{t} R(x(s)) d s,
\end{aligned}
$$


where

$$
\begin{aligned}
& Q(x, y):=\frac{\cos x+\cos (x(t-\tau(t)))+1 / 2 \cos y+1 / 2 \cos (y(t-\tau(t)))}{5+\cos ^{2} x+\cos ^{2} y+x^{2}(t-\tau(t))+y^{2}(t-\tau(t))}, \\
& R(x(s)):=\frac{\left|\left(9 x^{2}(s)-2\right) \cos 3 x(s)+\left(9 x^{2}(s)+2\right)\left(3 x(s) \sin 3 x(s)-2\left(9 x^{2}(s)-2\right)\right)\right|}{\left(9 x^{2}(s)+2\right)^{2}} .
\end{aligned}
$$

Comparing system (7) with system (59), we obtain the following relations. (i) The function

$$
f(\cdot):=3 y+\frac{\cos x+\cos (x(t-\tau(t)))+1 / 2 \cos y+1 / 2 \cos (y(t-\tau(t)))}{5+\cos ^{2} x+\cos ^{2} y+x^{2}(t-\tau(t))+y^{2}(t-\tau(t))} .
$$

Noting that

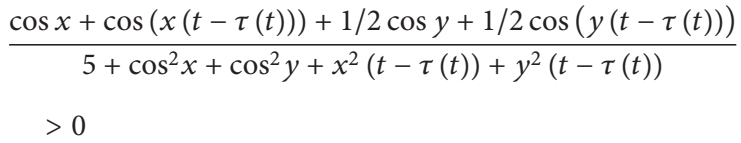

for all $t \geq 0, x$ and $y$, we assert that

$$
\frac{f(\cdot)}{y} \geq a=3
$$

for all $t \geq 0, x$ and $y \neq 0$. The function $f(\cdot)$ is shown in Figure 4.

(ii) The function

$$
g(x):=2 x+\frac{x \cos 3 x}{2+9 x^{2}} .
$$

Let

$$
G(x):=\frac{\cos 3 x}{2+9 x^{2}}
$$

Since

$$
-1 \leq \frac{\cos 3 x}{2+9 x^{2}} \leq 1
$$

for all $x$, it follows that

$$
1=b \leq \frac{g(x)}{x} \leq B=3
$$

for all $x \neq 0$.

(iii) Furthermore, from (ii) above we have

$$
\begin{aligned}
& \left|g^{\prime}(x)\right| \\
& :=\frac{\left|\left(9 x^{2}-2\right) \cos 3 x+\left(9 x^{2}+2\right)\left(3 x \sin 3 x-2\left(9 x^{2}-2\right)\right)\right|}{\left(9 x^{2}+2\right)^{2}} .
\end{aligned}
$$

It is not difficult to show that

$$
\left|g^{\prime}(x)\right| \leq L=\frac{5}{2}
$$

for all $x$. The functions $g(x) / x, G(x)$, and $\left|g^{\prime}(x)\right|$ are shown in Figure 5.

(iv) The function

$$
\phi(t):=\frac{5+2 t+\sin 6 t}{2+t^{2}}=2+\frac{1+\sin 6 t}{2+t^{2}} .
$$

Since

$$
\frac{1+\sin 6 t}{2+t^{2}}>0
$$

for $t \in[0,2 \pi]$, it follows that

$$
\phi(t)=\frac{5+2 t+\sin 6 t}{2+t^{2}} \geq \phi_{0}=2>1
$$

for all $t \geq 0$. The graph of function $\phi(t)$ is shown in Figure 6.

(v) Moreover, choose $\beta_{0}=1 / 2$ and from (i) to (iv) of Example 2 we have

$$
\alpha=\min \left\{\frac{6}{65}, \frac{1}{5}\right\}=\frac{6}{65},
$$

so that

$$
\tau(t) \leq \frac{6}{65} .
$$

(vi) The function

$$
\begin{aligned}
p(t, x, y) & :=\frac{1+x^{2}+y^{2}+2 \cos (x+y)}{2\left[1+x^{2}+y^{2}\right]} \\
& =\frac{1}{2}+\frac{\cos (x+y)}{1+x^{2}+y^{2}} .
\end{aligned}
$$




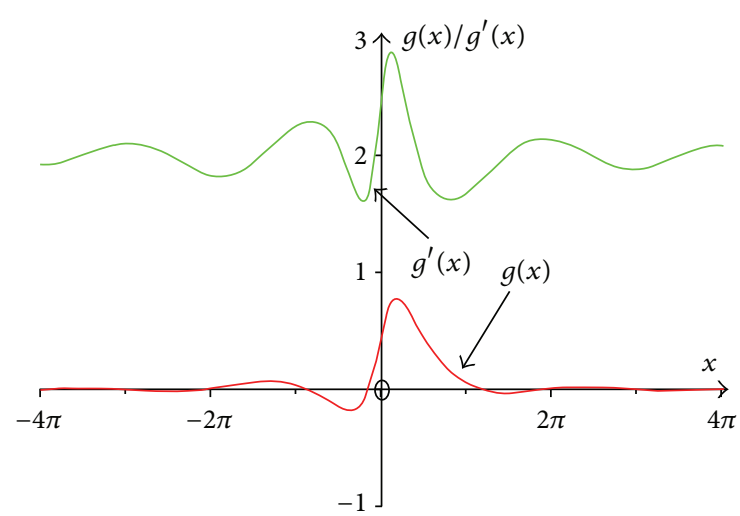

FIgURE 3: Functions $g(x)$ and $g^{\prime}(t)$.

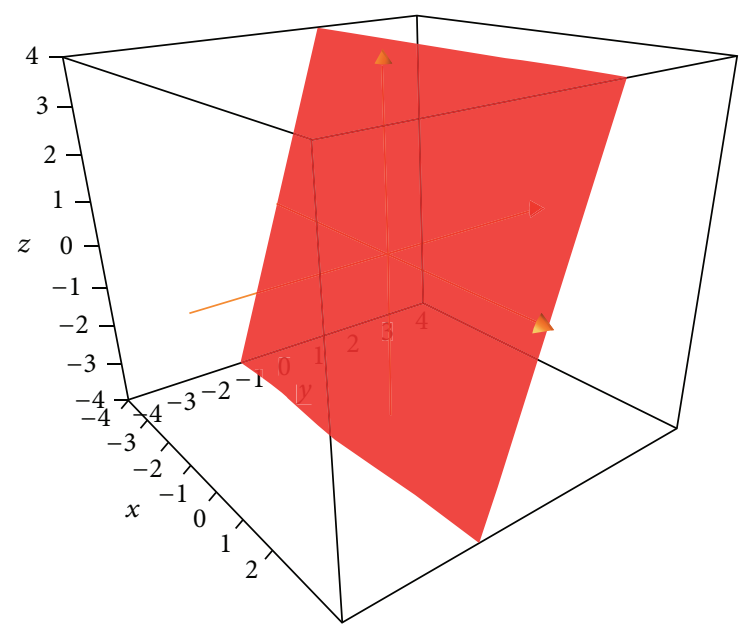

Figure 4: Function $f(\cdot)$.

Since

$$
\frac{\cos (x+y)}{1+x^{2}+y^{2}} \leq 1
$$

for all $x$ and $y$, it follows that

$$
|p(t, x, y)| \leq M=\frac{3}{2}
$$

for all $t \geq 0, x$ and $y$.

All hypotheses of Theorems 9 and 14 are satisfied; thus by Theorems 9 and 14 we have that

(i) solutions of system (59) are uniformly bounded and uniformly ultimately bounded;

(ii) there exists a unique periodic solution of period $\omega$,

respectively. If $p(t, x, \dot{x})=0$ and $p(t, x, y)=0$ in (57) and system (59), respectively, and conditions (i) to (v) of Example 2 hold, then hypotheses of Theorem 12 hold; hence by Theorem 12

(iii) the trivial solution of the system (59) (when $p(t, x, y)=0)$ is uniformly asymptotically stable.

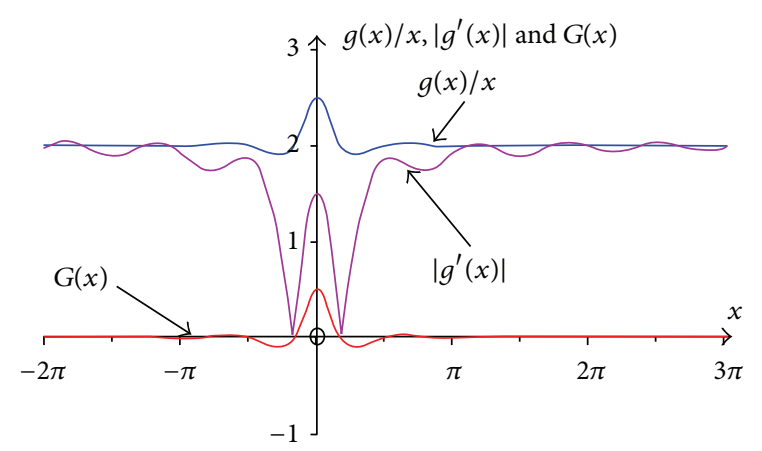

Figure 5: Functions $g(x) / x, G(x)$ and $\left|g^{\prime}(x)\right|$.

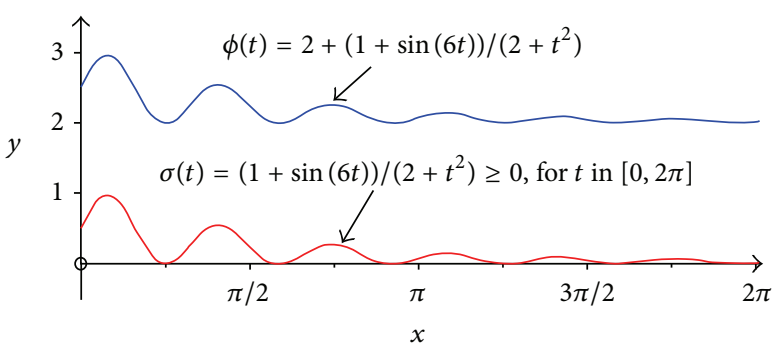

FIGURE 6: Function $\phi(t)$.

\section{Competing Interests}

The authors declare that they have no competing interests.

\section{References}

[1] T. A. Burton and L. Hatvani, "Asymptotic stability of second order ordinary, functional, and partial differential equations," Journal of Mathematical Analysis and Applications, vol. 176, no. 1, pp. 261-281, 1993.

[2] T. A. Burton, Stability and Periodic Solutions of Ordinary and Functional Differential Equations, Mathematics in Science and Engineering, Academic Press, Orlando, Fla, USA, 1985.

[3] T. A. Burton, Volterra Integral and Differential Equations, Academic Press, New York, NY, USA, 1983.

[4] J. K. Hale, Theory of Functional Differential Equations, Springer, New York, NY, USA, 1977.

[5] T. Yoshizawa, "Liapunov's function and boundedness of solutions," Funkcialaj Ekvacioj, vol. 2, pp. 71-103, 1958.

[6] T. Yoshizawa, Stability Theory and Existence of Periodic Solutions and Almost Periodic Solutions, Springer, New York, NY, USA, 1975.

[7] T. Yoshizawa, Stability Theory by Liapunov's Second Method, The Mathematical Society of Japan, 1966.

[8] A. T. Ademola, "Boundedness and stability of solutions to certain second order differential equations," Differential Equations and Control Processes, no. 3, pp. 38-50, 2015.

[9] J. G. Alaba and B. S. Ogundare, "On stability and boundedness properties of solutions of certain second order nonautonomous nonlinear ordinary differential equation," Kragujevac Journal of Mathematics, vol. 39, no. 2, pp. 255-266, 2015.

[10] B. Cahlon and D. Schmidt, "Stability criteria for certain secondorder delay differential equations with mixed coefficients," 
Journal of Computational and Applied Mathematics, vol. 170, no. 1, pp. 79-102, 2004.

[11] A. Domoshnitsky, "Nonoscillation, maximum principles, and exponential stability of second order delay differential equations without damping term," Journal of Inequalities and Applications, vol. 2014, article 361, 2014.

[12] G. A. Grigoryan, "Boundedness and stability criteria for linear ordinary differential equations of the second order," Russian Mathematics, vol. 57, no. 12, pp. 8-15, 2013.

[13] Z. Jin and L. Zengrong, "On the global asymptotic behavior of solutions to a non autonomous generalized Liénard system," Journal of Mathematical Research and Exposition, vol. 21, no. 3, pp. 410-414, 2001.

[14] A. J. Kroopnick, "Bounded solutions to $x^{\prime \prime}+q(t) b(x)=f(t)$," International Journal of Mathematical Education in Science and Technology, vol. 41, no. 6, pp. 829-836, 2010.

[15] B. S. Ogundare and A. U. Afuwape, "Boundedness and stability properties of solutions of generalized Liénard equations," Kochi Journal of Mathematics, vol. 9, pp. 97-108, 2014.

[16] S. B. Ogundare and G. E. Okecha, "Boundedness, periodicity and stability of solution $\dot{x}+a(t) g(\dot{x})+b+(t) h(x)=p(t ; x, x)$," Mathematical Sciences Research Journal, vol. 11, no. 5, pp. 432443, 2007.

[17] C. Tunç, "A note on the bounded solutions to $x^{\prime \prime}+c\left(t, x, x^{\prime}\right)+$ $q(t) b(x)=f(t)$," Applied Mathematics \& Information Sciences, vol. 8, no. 1, pp. 393-399, 2014.

[18] C. Tunç, "Boundedness analysis for certain two-dimensional differential systems via a Lyapunov approach," Bulletin Mathematique de la Societe des Sciences Mathematiques de Roumanie, vol. 53, no. 1, pp. 61-68, 2010.

[19] C. Tunç, "New results on the existence of periodic solutions for Rayleigh equation with state-dependent delay," Journal of Mathematical and Fundamental Sciences, vol. 45, no. 2, pp. 154162, 2013.

[20] C. Tunç, "Stability and boundedness in multi delay vector Liénard equation," Filomat, vol. 27, no. 3, pp. 435-445, 2013.

[21] C. Tunç, "Stability and boundedness of solutions of nonautonomous differential equations of second order," Journal of Computational Analysis and Applications, vol. 13, no. 6, pp. 1067-1074, 2011.

[22] F. Wang and H. Zhu, "Existence, uniqueness and stability of periodic solutions of a duffing equation under periodic and anti-periodic eigenvalues conditions," Taiwanese Journal of Mathematics, vol. 19, no. 5, pp. 1457-1468, 2015.

[23] Z. Xianfeng and J. Wei, "Stability and boundedness of a retarded Liénard-type equation," Chinese Quarterly Journal of Mathematics, vol. 18, no. 1, pp. 7-12, 2003.

[24] A. F. Yeniçerioğlu, "The behavior of solutions of second order delay differential equations," Journal of Mathematical Analysis and Applications, vol. 332, no. 2, pp. 1278-1290, 2007.

[25] A. F. Yeniçerioğlu, "Stability properties of second order delay integro-differential equations," Computers and Mathematics with Applications, vol. 56, no. 12, pp. 3109-3117, 2008.

[26] Y. F. Zhu, "On stability, boundedness and existence of periodic solution of a kind of third order nonlinear delay differential system," Annals of Differential Equations, vol. 8, no. 2, pp. 249259, 1992. 


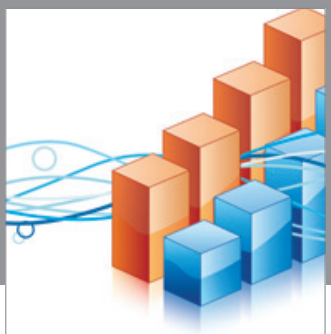

Advances in

Operations Research

vatem alat4

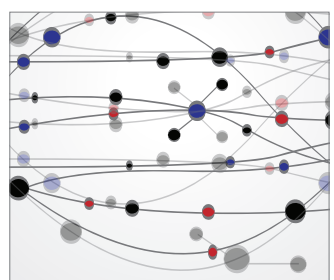

\section{The Scientific} World Journal
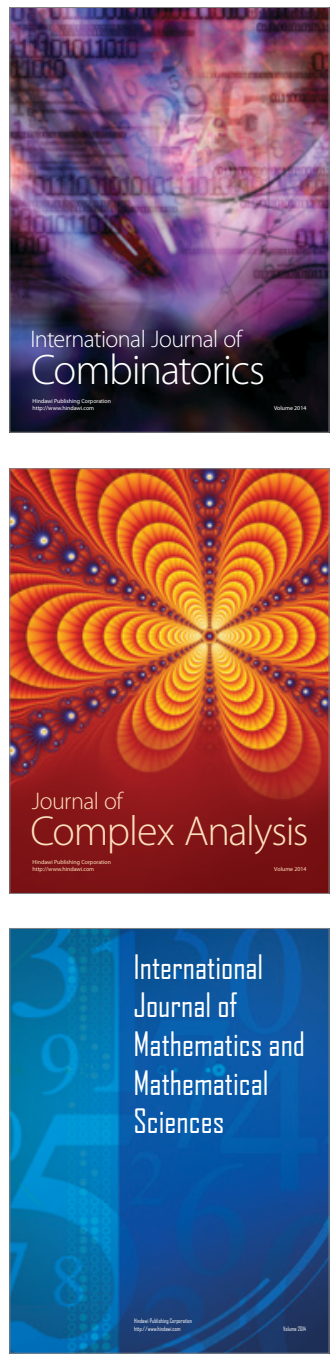
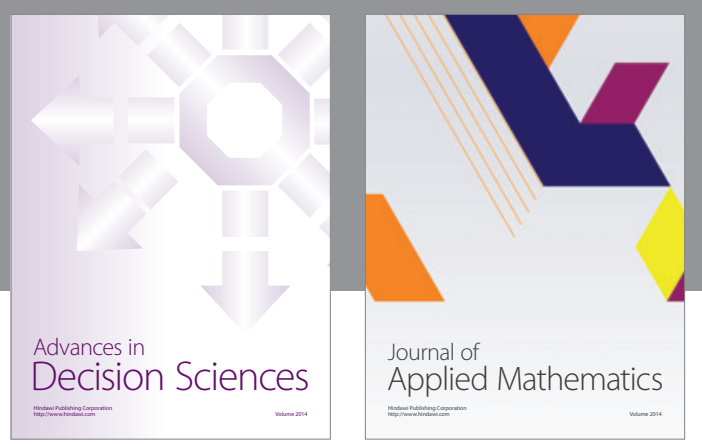

Algebra

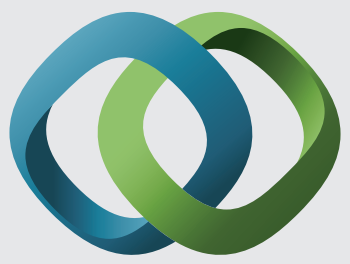

\section{Hindawi}

Submit your manuscripts at

http://www.hindawi.com
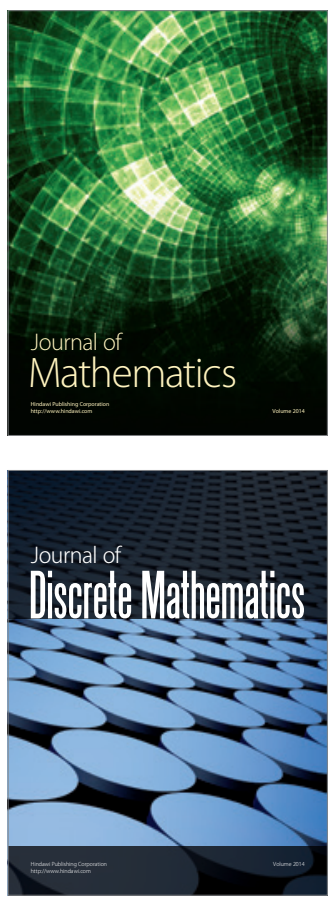

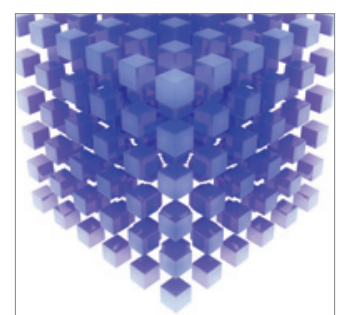

Mathematical Problems in Engineering
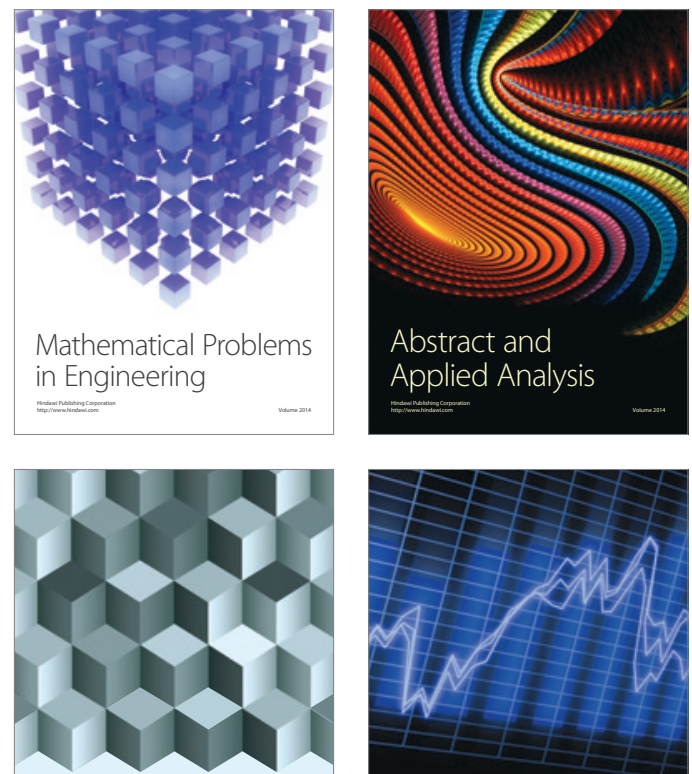

Journal of

Function Spaces

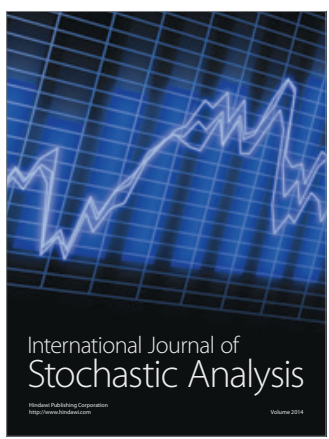

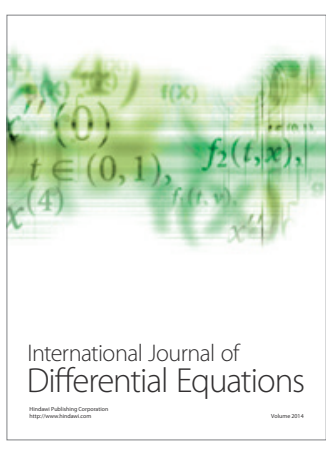
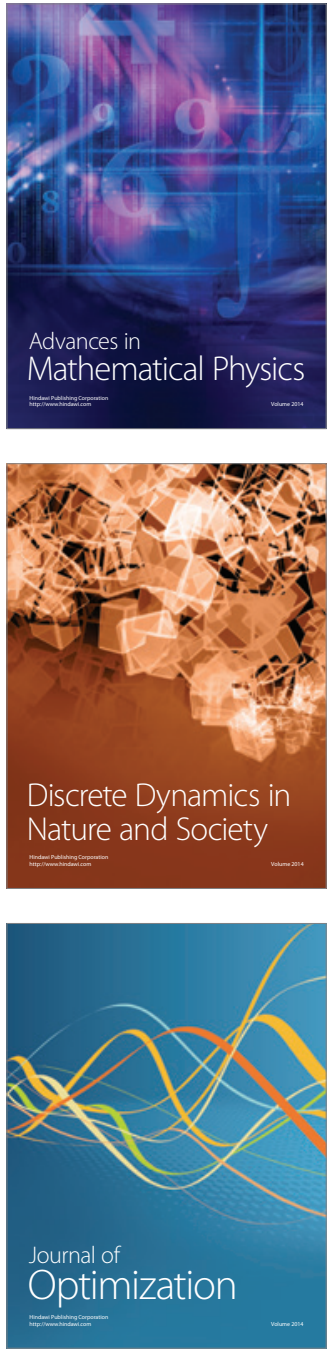